\title{
Solid pseudopapillary tumour of pancreas: A rare neoplasm
}

\author{
Miglani Ripan K. • Nagesh N. S. • Murthy Divakar S. • Bhat Ravi Shankar • Nayak Raj Sekhar • Ashok Kumar K. V.
}

Received: 13 August 2008 / Accepted: 21 October 2008

(C) Association of Surgeons of India 2010

\begin{abstract}
Solid pseudopapillary tumour (SPT) is the rare tumour of pancreas with unknown aetiology and good prognosis. Occurs predominantly in young women of reproductive age group. Not many cases have been reported from India. We report a case of a young woman who presented with persistent back pain having large tumour of body of pancreas treated successfully by left pancreatectomy.
\end{abstract}

Keywords Frantz tumour · Solid pseudopapillary tumour of pancreas $\cdot$ Pancreatic pseudotumour
Miglani Ripan K. · Nagesh N. S. · Murthy Divakar S.

B. R. Shankar · N. R. Sekhar · Ashok Kumar K. V.

Department of Surgical Gastroenterology,

Bangalore Medical College and Research Institute, India

Miglani Ripan K. ( $\square)$

E-mail: ripanmiglani@yahoo.com

\section{Introduction}

Solid pseudopapillary tumour (SPT) of the pancreas is a rare neoplasm accounting for $0.13-2.7 \%$ of all pancreatic tumour. It occurs almost exclusively in young women of reproductive age group and is associated with increased progesterone levels which is being thought of as the reason for this. It is also known as solid and papillary epithelial neoplasm, papillarycystic neoplasm and cystic-solid papillary carcinoma of the pancreas. SPT is thought to have a benign course in most of cases although it can be malignant in $15 \%$. On account of the scant literature on the subject and very few case reports especially from India, we considered it interesting to contribute to the case histories with clinical case of a 23-year-old woman.

\section{Case report}

A 23-year-old married woman presented to us with history of persistent back pain since 6 years. Examination was non-contributory except for mild tenderness in left hypochondrium. U/S suspected a large mass in the region of body of pancreas, contrast enhanced computed tomogram (CECT) abdomen (Fig. 1) done which showed a $7 \times 5 \mathrm{~cm}$ lesion in the body and the tail of pancreas with solid and cystic components in it. Lesion seemed to be free from splenic and superior mesenteric vessels. Her haemogram, liver function tests (LFTs), serum amylase and CA19-9 were within normal limits. Patient was taken up for laparoscopic left pancreatectomy. On laparoscopy large exophytic mass of dimensions $10 \times 8 \mathrm{~cm}$ was seen which was extending up to superior mesenteric vein (SMV). Conversion to laparotomy was done through midline incision and tunnel over SMV was made followed by left pancreatectomy 


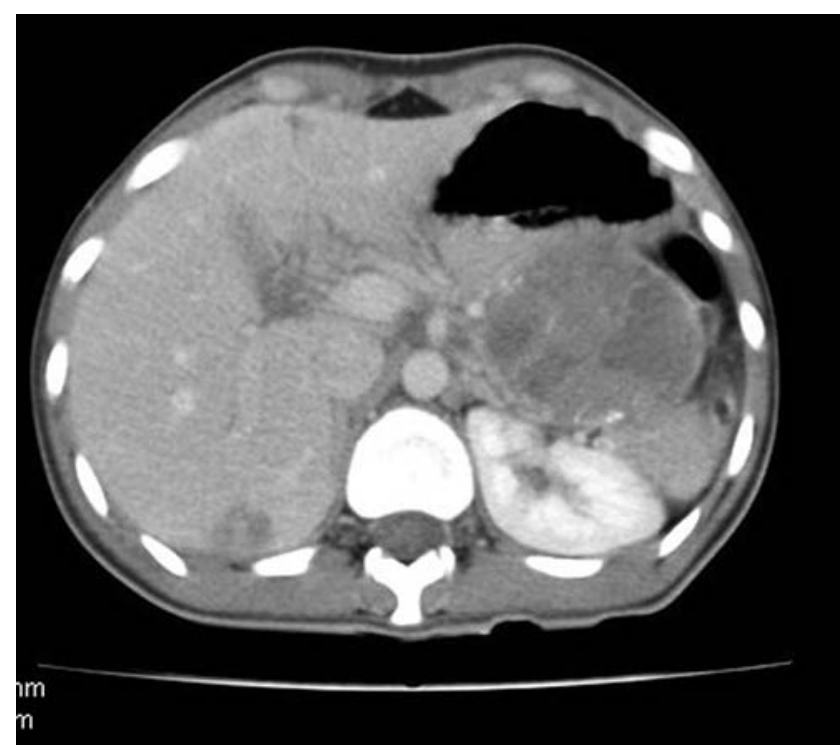

Fig. 1 CECT abdomen showing tumour in the body of pancreas

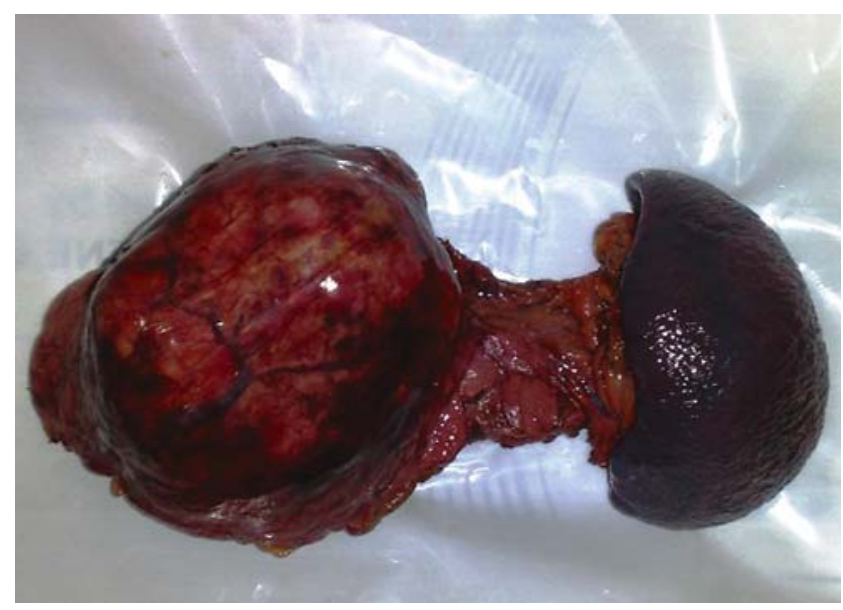

Fig. 2 Gross specimen of the tumour

with splenectomy was done. Postoperative period was uneventful and patient did not develop any endocrine or exocrine insufficiency.

Histopathological evaluation (Fig. 2) showed $7 \times 4 \mathrm{~cm}$ cystic lesion showing areas of haemorrhage and necrosis.

Microscopically it showed solid and papillary components with solid areas showing sheats of monomorphic cells with papillae lined by multiple layers of cells. Cells showed no significant pleomorphism or atypia. At places there was abundant mucin secretion, resected margins were negative for the tumour.

\section{Discussion}

Although it was first described by Frantz in 1959 [1], SPT has only recently been characterised as a clinical entity due to improved diagnostic techniques and a detailed pathological description, new cases are being recognised frequently. The medical literature counts almost 500 cases, most of which identified in the last 25 years [2]. Synonyms include solid and cystic tumour, solid and papillary epithelial neoplasm, papillarycystic neoplasm, papillary cystic epithelial neoplasm, papillarycystic tumour, and Franz tumour. In 1996, the World Health Organization (WHO) renamed this tumour as SPT for the international histologic classification of tumour of the exocrine pancreas. It is neoplasm of the pancreas with low malignancy. Its histogenesis is still unknown. Male-tofemale ratio of about 1:10-1:15 (age range 11-69 years). The higher incidence in young women leads us to assume that it originates from pluripotential primitive cells of a blastomatous nature.

Progesterone receptors are found positive, although not in all cases; estrogen receptors are negative [3]. Pregnancy determines a powerful augmentative stimulus through the secretion of progesterones [4]. Eighty to $85 \%$ cases it runs benign course while in about $15 \%$ can be malignant.

Macroscopically the tumour generally appears as a mass of variable size, well encapsulated with ares of haemorrhage, necrosis and cystic change. Microscopically, the tumour cells have a cylindrical, cuboidal or polygonal shape and form solid cystic or papillary areas. Hallmark histologic pattern occurs when the tumour cells form papillary configurations composed of a fibrovascular stalk surrounded by several layers of epithelial cells.

Mitosis is quite uncommon, and this confirms the low grade of malignancy. Immunohistochemically, pancreatic SPT is positive for vimentin, neuron specific enolase (NSE) and $\alpha 1$-antitrypsin [5]. These immunohistochemical characteristics distinguish it from the non-functioning pancreatic neuroendocrine tumour. SPT can develop at any site; both the head and body tail of the pancreas may be involved, although manifestations in the body tail are slightly predominant.

Scattered forms are rare. Clinically, SPT mostly manifests as an abdominal mass that progressively increases in size, occasionally with continuous pain in the epigastric region or going to back. About two-third of the patients are symptomatic. Rarely severe pain due to rupture of the tumour and development of a haemoperitoneum [6].

Age $>40$, male gender, septations and multilocularity should raise suspicion of malignant SPT.

Diagnostic imaging generally shows a pancreatic mass of a cystic nature with peripheral calcifications.

The preferred treatment is surgical removal of the tumour, which is curative in most cases. It is, however, recommended to make sure that the resection margins are tumour free. Although reports of treating SPT of head only by enucleation without recurrences are there [7].

Local recurrence and distant metastasis are uncommon but may occur in the regional lymph nodes, liver and 
peritoneum. In such cases, an aggressive surgical approach may still be curative [8].

Radiotherapy, chemotherapy, hormonal therapy all have been used in few cases but substantial data on their efficacy is lacking.

\section{Conclusion}

SPT is a rare form of pancreatic tumour. Differentiating it form adenocarcinoma of pancreas is important because of different natural history and prognosis. Surgery is generally curative. With ongoing research etiology of SPT should be clearer in near future.

\section{References}

1. Frantz VK (1959) Tumours of the pancreas. In: Atlas of Tumour Pathology. Armed Forces Institute of Pathology, Washington, DC; pp 32-33

2. Marrano D, Casadei R, Santini D (2001) Tumouri cistici del pancreas. Piccin Nuova Libraria, Padua, Italy

3. Morales A, Ruiz Molina JM, Esteves HO, Robles-Diaz G, Diaz-Sanchez V (1998) Papillary-cystic neoplasm of the pancreas. A sexsteroid dependent tumour. Int J Pancreatol 24:219-225

4. Ganepola GA, Gritsman AY, Asimakopulos N, YiengprukSawan A (1999) Are pancreatic tumours hormone dependent? A case report of unusual rapidly growing pancreatic tumour during pregnancy, its possible relationship to female sex hormones, and review of the literature. Am Surg 65:105-111

5. Pettinato G, Manivel SC, Ravetto C, Terracciano ML, Gould EW, Di Tuoro A, Jaszcz W, Albores-Saavedra J (1992) Papillary cystic tumour of the pancreas. A clinicopathologic study of 20 cases with cytologic, immunochemical, ultrastructural and flow cytometric observations and a review of the literature. Am J Clin Pathol 98:478-488

6. Jeng LBB, Chen MF, Tang RP (1993) Solid and papillary neoplasm of the pancreas. Emphasis on surgical treatment. Arch Surg 128:433-436

7. Akiama H, Ono K, Takano M, Sunida K, Ikuta K, Miyamoto O (2002) Solid pseudopapillary tumour of pancreatic head causing marked distal atrophy: A tumour originated posterior to main pancreatic duct. Int J Gastrointest Cancer 32:47-52

8. Freda F, Nunziata L, Antropoli M, D'Amodio AS, Manganiello A, Petronella P (2004) Outcome of surgical treatment of carcinoma of the pancreas. Tumouri 90:27-31 